\title{
A Unique Six Sigma Based Segmentation Technique for Brain Tumor Detection and Classification using Hybrid CNN-SVM Model
}

\author{
Arati Kothari, B. Indira
}

\begin{abstract}
An intelligent organizing scheme to detect and classify normal, abnormal MRI brain sequences has been illustrated here. At present, handling of brain tumors disease and decision is based on radiological appearance and its symptoms. Magnetic-Resonance-Imaging (MRI) is a powerful substantial precise instrument for functional conclusion of brain tumorous. In existing study, broad range of methods is used for brain cancer detection and classification. Under this methods viz., image pre-processing, enhancement, segmentation, feature mining and resulting classification is efficiently conducted. Furthermore, when various machine learning algorithms like: Six Sigma, Convolutional Neural Network (CNN), Support Vector Machine (SVM), are employed to detect and extract the tumor region and classify numerous sequence of imageries, it is witnessed from our results that this Hybrid CNN-SVM model gives maximum classification accuracy rate of $99.33 \%$ compared to previous models. The foremost aim of this research is to get an effective result for detecting type of brain tumor using six sigma based segmentation technique, and to achieve efficient classification rate, using hybrid CNN-SVM model.
\end{abstract}

Index Terms: Classification, CNN, Hybrid CNN-SVM, MRI, Six Sigma Segmentation, SVM, Tumor.

\section{INTRODUCTION}

Brain is the most composite part in human body which has billions of cubicles. A tumor is formed if there are unrestrained separating cells creating an irregular group of cells inside the brain [2]. Every year worldwide, 12.7 million people getting diagnosed with brain diseases. A tumor is anomalous cells development inside the brain. Tumorous can be malignant (cancerous) or benign (noncancerous) [3]. Compared to usual cells, cancer cells concerns about abandoned cell growth and can grow to adjacent tissue. Even though benign tumor can grow bigger and damage healthy tissues and organs that cause hypothetically disturbance to their functioning, they rarely conquer other tissue [4].

Revised Manuscript Received on 30 July 2019.

* Correspondence Author

Arati Kothari*, Department of Computer Science, Gulbarga University, Kalaburagi, India.

Dr. B. Indira, Department of Computer Science, Chaitanya Bharathi Institute of Technology, Hyderabad, India.

(c) The Authors. Published by Blue Eyes Intelligence Engineering and Sciences Publication (BEIESP). This is an open access article under the CC-BY-NC-ND license http://creativecommons.org/licenses/by-nc-nd/4.0/
A foremost tumor begins from brain itself, whereas inferior brain tumorous (i.e. metastatic tumorous) initiates from other parts in body. The proposed design comprises following modules: pre-processing, feature mining (extraction), and training with classification. Pre-processing will be applied to give better superiority of image brain sequences.

Medical images sequences are despoiled from various types of noises. It is much essential to get better quality of brain sequences for precise interpretations for given domain application. Feature mining denotes extraction of number of reckonable measurements of image sequences basically utilized for pathology decision making related to tissue structure. Renovating input set of data to set of comparative textures is known as extraction of features. Choosing features is an important processing stage in identification and classification of issues, and it can applicable efficiently to several fields.Classifiers like, SVM, KNN, CNN, Probabilistic Neural Network (PNN), Artificial Neural Network (ANN), Hidden Markov Model (HMM), etc. are utilized in diverse real time applications. Every single classification algorithms named before has its own associated and distinct properties along with drawbacks. In KNN, foremost drawback is that it makes use of all features which is computationally difficult, when size of training set raises. Alongside, precision of $\mathrm{k}$-nearest neighbor classifier is brutally corrupted through existence of noisy and dissimilar features, mainly while number of attributes increases. In PNN, drawback is compared to MP (multilayer perceptron) networks, it is slower than and it demands extra memory space. ANN gives better classification outcomes than others with higher dimensional features. However, memory usage and high computing cost is the foremost drawback of ANN. Bayesian model performs in lowest computational cost but it is less precise than the traditional method such as $\mathrm{CNN}$.

\section{LITERATURE SURVEY}

The literature survey of the proposed work setup on research carried out by many researches related to classification brain tumor.

Tanvi Gupta et.al [01] has evaluated its utility for rapid diagnosis and accuracy of intellectual tumors. 200 themes were categorized into usual and unusual by employing FLAIR acquirement. The sequences are standardized to get 12 beneficial pieces to be measured as patient feature map for grade. 


\section{An Unique Six Sigma Based Segmentation Technique for Brain Tumor Detection and Classification using Hybrid CNN-SVM Model}

DWT is employed for mining of features and PCA is taken for selection of mined features. SVM (Support-Vector-Machine), CART (Classification and Regression Tree), k-NN (k-Nearest Neighbor), and RF (Random forest) classifiers are verified. Conducting K-fold cross authentication in every train-test ratios, they acquired ceiling level classification accuracy with intelligible specificity and sensitivity utilizing only linear SVM.

Heba Mohsen et.al [02] has told that DL (Deep learning) is a novel ML (machine learning) area which attained a lot more attention in recent years. It was broadly realistic to numerous solicitations and demonstrated to be an efficient ML device for several of challenging difficulties. Here they have utilized DNN classifier which is one of the major DL architectures for organizing a dataset of 66 brain MRIs into 4 types e.g. normal, glioblastoma sarcoma, and metastatic-bronchogenic-carcinoma tumorous. This will be later concatenated with DWT (discrete wavelet transform) a dominant feature mining utensil and PCA (principal components analysis) for choosing relative features.

Mohammad Havaei et.al [03] has investigated a model for interactive tumor segmentation that, at its core, treats difficulties of collaborating segmentation as a ML issue. This technique has a benefit over traditional ML techniques for task where generalization is done through brains. The issue is that they want to deal with intensity bias correction and other MRI-specic noise. Here they avoid these issues by approaching problem inside brain overview. Particularly, they illustrated a semi-automatic model that segments tumor through training and simplifying inside that brain only, based on some least user interaction.

Praveen G.B et.al [04] has suggested a hybrid approach to brain tumor detection and classification through magnetic resonance imageries. Initial it deals with pre-processing that includes skull detection, noise filtering, etc. The next stage deals with feature mining of MRI brain sequences by GLCM. Further phase contracts with classification of abnormal, normal by taking Least-Squares SVM classifier with MP (Multilayer perceptron) kernel. Lastly segmentation stage of tumor part is performed from brain employing fast bounding-box. The tests were demonstrated out on 100 imageries having of 25 normal and 75 abnormal.

Vijay Wasule et.al [05] told that classification of brain sequences is a crucial task. A model to organize tumor into benign vs. malignant, is depicted. This technique picks GLCM approach for feature extraction from image sequence and stores as a feature vector. These mined features were classified using KNN and supervised SVM techniques. The derived architecture is conducted on 80 images of brats 2012 training database and 251 images (166 benign and 85 malignant) of clinical brain database.

Hari Babu Nandpuru et.al [06] has depicted an intellectual classification scheme to identify normal, abnormal MRI brain sample. The significant mode of identify the human disease in medical image like MRI, ECG and CT-scan are proficiently. To overcome human error, an automatic intellectual classification model is illustrated that caters need for classification of sample. MRI technique is employed to study of human brain. Here SVM is utilized for classification and texture and gray scale features are employed for feature mining.

Khalid Usman et.al [07] has suggested a tumor classification and segmentation technique for multi-modality MRI scans. The sequence data from multimodal tumor segmentation test are used that are recorded and skulls tripped, and histogram-matching is accomplished with respect to high volume contrast. From pre-processed sequences, features are then mined: intensity, wavelet texture and local neighborhood intensity differences. The combined feature statistics are consequently given to RF classifier to forecast 5 categories: background, necrosis, edema, non-enhancing tumor and enhancing tumor.

A. Rathna Raju et.al [08] has suggested an automated technique to train multi- SVNN classifier for classification by utilizing Harmony-Crow Search (HCS) Optimization technique. The tumor segmentation is implemented utilizing Bayesian-fuzzy-clustering method; however classification of tumor is achieved by employing suggested HCS Optimization technique based on multi-SVNN classifier. The awaited technique of classification describes brain tumor level employing features of segments produced based on Bayesian fuzzy clustering. The vigorous features are acquired using scattering transform, information theoretic measures, and wavelet transform.

Gopalakrishnan Sethumadhavan et.al [09] has presented an analysis model for deviations of RGB spectrum of lesion skin picture sequences employing a new segmentation practice taken on Six Sigma theory. This examines further anticipates on propagation and incidence of cancer. It is built on fundamental principles of control Charts which make attentions on fact that changeability is not exist in all repetitive procedures. Assorted color deviation inside skin is reserved as a transferable cause and is because of fact emission of over melanin. These variations retain higher magnitude paralleled to chances of causes due to color deviations originate in normal skins.

Ajit Danti et.al [10] has given a new scheme for arecanut colour based classification into 2 classes. The suggested scheme has 3 steps: (i) Segmentation; (ii) Masking; and finally, (iii) classification. The RGB sequence is altered into YCbCr color space. 3 sigma control limits are exploited for $\mathrm{YCbCr}$ image for effectual segmentation of arecanuts. Arecanut color space is demonstrated by 3 sigma control limits, in which it insurances more significant deviations of colour components of arecanut. The upper, lower bounds of color components are utilized to segment arecanut effectually. Classification is finished based on green and red color components of segmented region of arecanuts.

\section{PROPOSED METHODOLOGY}

The detailed methodology and process of the proposed exudates detection is been described in the below block diagram. The architecture is divided into two separate parts viz, testing and training. The brain MRI image will be taken as input. 
The input image will be pre-processed using histogram equalization followed by segmentation using six sigma techniques. The various features of brain MRI image will be extracted and tracked by convolution neural network (CNN) feature extraction process. These features will be matched with already extracted features during training phase based on which the type of tumor will be classified using support vector machine (SVM) classifier.

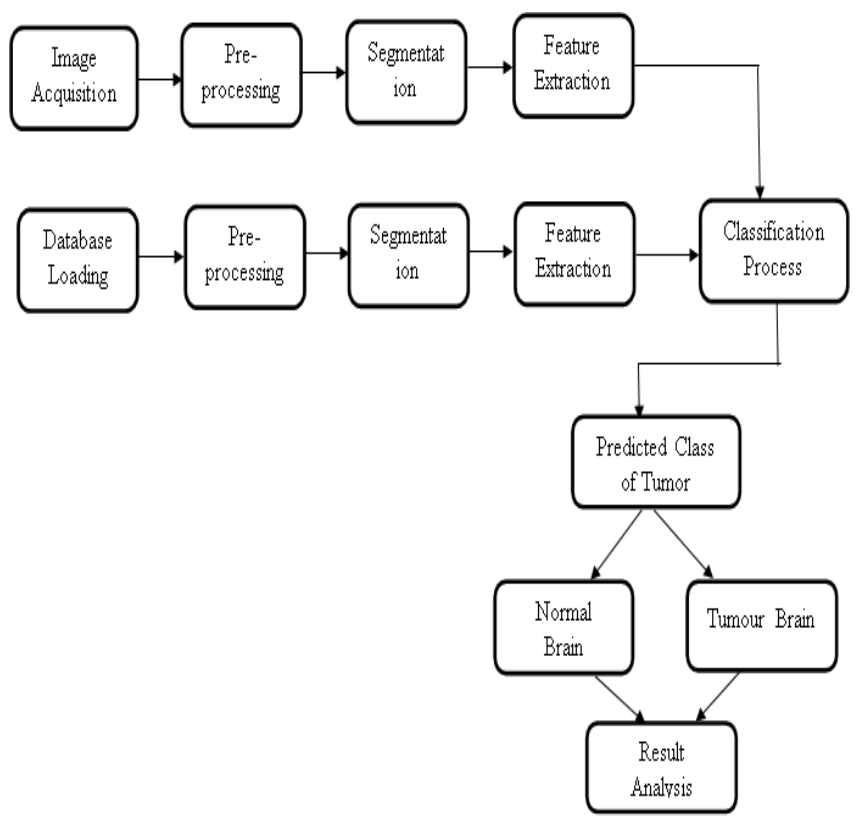

Figure 1: Classification of Brain Tumor.

A.Pre-Processing: The input MRI image might be affected by several noises while image generation. Likewise, patient movement can be reason various noises. The noise elimination is a crucial task to get more accurate segmentation result. The input color image will first be converted into grayscale image. Further it undergoes histogram equalization for contrast adjustment as it shows the distribution of the pixel values above color bar of the color map. Through this intensity will be even better dispersed on histogram. This permits for lower local contrast areas to acquire a higher contrast.

B.Six Sigma Segmentation: Countless practical segmentation complications need higher statistics than confined in single spectral band. Color sequences are regular example, in which statistical data has been coded in 3 bands. Generally multi-spectral threshold segmentation technique is obtained by deriving thresholds individually in every band spectral are joined to one single segmented part.

[1] Six Sigma Thresholding on Images: RGB color plane utilized as illustrations of pixel values is chosen for examination. Data extracted by control charts rests on basis of assortment of sub-groups with max uniformity. To assist this, image sequence of dimension $\mathrm{Mx} \mathrm{N}$ is cut into minor small regions of $m \times n$ sizes, here, $m=n=4,5$ are taken as trials of size $m^{*} n$ each. Here, $M * N / m * n$ samples are ordered in column majoring. To plot control-charts, $\pm 3 \sigma$ bounds are derived and then are categorized as bound limits. Assignable reasons of deviations will be determined by way of deriving sub sections falls outside bound limits on $\mathrm{X}$ or $\mathrm{R}$ chart. Subsiding of all other small sections, within boundary limits denotes scattering of layer is in controller [10, 11]. A deviation in color broadcast is inspected via taking small regions that drops outside bound limits. The pixels owned by these sub regions, that fall outside bound limits in every slicing and falls on these common connections among various slicing are recognized.

[2] Intersection Regions: Here, numerous prospects of joining sub regions, that fall exterior to bound limits due to (4 $\mathrm{x} 4),(4 \times 5),(5 \times 4)$ and $(5 \times 5)$ slicing are described. It is renowned that pixel $(\mathrm{x} \mathrm{i}, \mathrm{j})$ due to Category 2 is well important because of exceptionality $[16,17]$. The lower the window size, higher will be accuracy.

C.Feature Extraction: Convolutional Neural Network feature extractor trained with image sequence training dataset, of which certain portion was selected arbitrarily as validation set while training. Especially face of neural network comprised of 3 consecutive convolutional layers including 8 , $16,32,64$ filters in every layer correspondingly. Convolutional filter size for every layer is [4 4] and 2x2 max-pooling is conducted next to each layer.Rectified linear units are exploited as activation function for convolutional neurons. Every output of this layer was batch regularized to stop neuron saturation in further layers. Figure 2 shows chart for CNN Specification.

14x1 Layer array with layers:

\begin{tabular}{|c|c|c|}
\hline 11 & Image Input & $256 \times 256 x 1$ images with 'zerocenter' normalization \\
\hline 11 & Convolution & 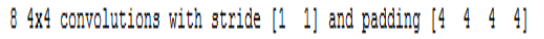 \\
\hline 11 & ReiJ & ReiJ \\
\hline 11 & Max Pooling & 4x4 max pooling with stride [ [2 2] and padding $\left[\begin{array}{llll}0 & 0 & 0 & 0\end{array}\right]$ \\
\hline 11 & Convolution & 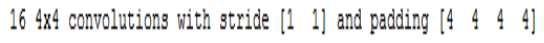 \\
\hline 11 & ReiJ & ReiJ \\
\hline 11 & Max Pooling & 4x4 max pooling with stride [ [2 2] and padding $\left[\begin{array}{llll}0 & 0 & 0 & 0\end{array}\right]$ \\
\hline 11 & Convolution & 32 4x4 convolutions with stride [1 1 1] and padding [ [ [ lllll] \\
\hline 11 & ReiJ & ReiJ \\
\hline 11 & Nax Pooling & 4x4 max pooling with stride $\left[\begin{array}{ll}2 & 2\end{array}\right]$ and padding $\left[\begin{array}{llll}0 & 0 & 0 & 0\end{array}\right]$ \\
\hline 11 & Convolution & 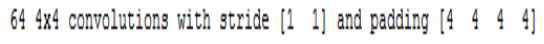 \\
\hline 11 & Fully Connected & 5 fully connected layer. \\
\hline 11 & Softmax & softmax \\
\hline 11 & Classification Output & crossentropyex \\
\hline
\end{tabular}

Figure 2: CNN Specifications.

The concluding area of network comprising of 2 fully-connected layers includes 512, with 16 neurons in every activation equation. The concluding output layer comprising of 4 neurons including soft-max activations for allocating a probability of prediction to every class. The network is trained by employing stochastic gradient descent to minimize cross- classification entropy loss, $70 \%$ imageries were utilized per training batch to avoid local minima and to raise training speed. 10 training epochs were conducted; imageries must be trained on multiple times to guarantee that network joins fully, specifically when training statistics are limited.

This was utilized to output transitional layer values, giving a 16 uncertain feature set for every input image. Feature sets were created for both the testing and training datasets to utilize in soft sensor training. [12].

D.Support Vector Machine Classification: SVM technique initially urbanized in 1963 by Lerner and Vapnik. SVM is dual classifier established on organized ML that gives proficient effect than previous techniques. 
SVM categorizes among 2 classes by building one hyper-plane in higher-dimensional space that can be operated for classification [6].

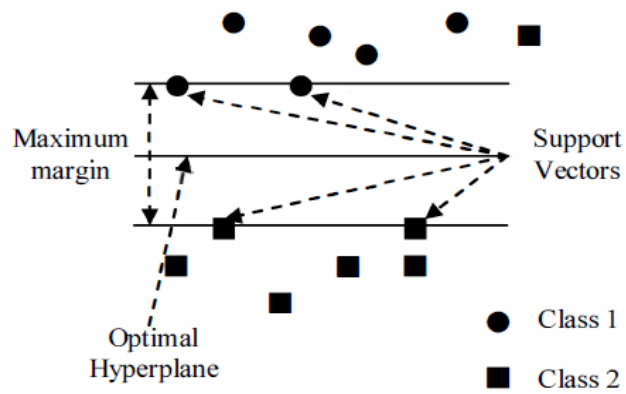

Figure 3: Linear and Non-Linear SVM Classification

A linear function of the form is given below

$$
f(x)=w^{5} x+b
$$

Such that for each training $x_{i}$ function produces $f\left(x_{i}\right) \geq 0$ for $\mathbb{Y}_{\mathrm{i}}=+1$ and $\mathrm{f}\left(\mathrm{x}_{\mathrm{i}}\right)<0$ for $\mathrm{y}_{\mathrm{i}}=-1$. In further words, training samples of 2 dissimilar classes are divided by hyper planef( $x)=w^{2} x+b=0$, where $w$ is weight vector and normal to hyper-plane, $\mathrm{b}$ is bias or threshold and $\mathrm{x}$, is data point. In Figure 3, it shows hyper-plane linear and non-linear classification of data. In our experiment RBF kernel is used in order to map non-linear samples to a high dimensional space, so it is different linear kernel can handle case when relation among attributes and class labels is nonlinear. Moreover, linear kernel is a distinct case of RBF since linear kernel with a penalty. Parameter has similar performance as RBF kernel with several parameters $(\mathrm{C}, \gamma)$. In addition, sigmoid kernel acts like RBF for certain parameters. There are 2 factors for RBF kernel: $\gamma$ and C. It is unknown earlier which $\gamma$ and $\mathrm{C}$ is finest for given issue; subsequently, several model assortments have to be done. Here aim is to detect $\operatorname{good}(\gamma, \mathrm{C})$ so that classifier can precisely forecast indefinite data. As deliberated above, a common tactic is to detach data set into 2 portions, of which one is considered unknown. The prediction accuracy obtained from "unknown" set more precisely reflects its performance on classifying an independent data set. An improved version of this procedure is known as cross validation.

\section{EXPERIMENTAL RESULTS}

The experimental results of the proposed model has illustrated here. In the proposed work medical MRI image dataset has been taken from MRMC (Mahadevappa Rampure Medical College, Department of Radiology). The brain MRI image is processed through four main steps: Image Pre-processing, Image Segmentation, Feature Extraction and Classification. The related results are shown below.

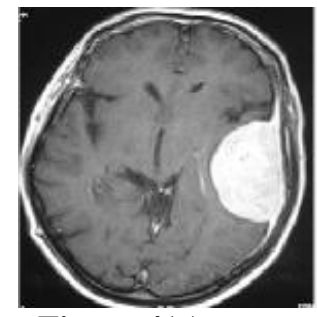

Figure 4(a)

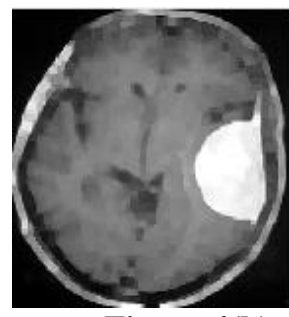

Figure 4(b)

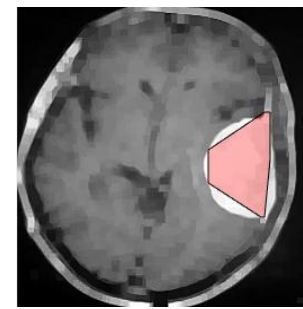

Figure 4(c)
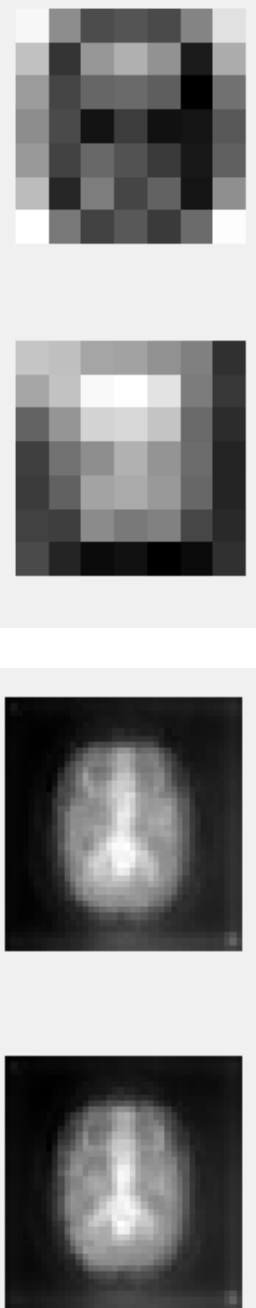

Figure 4(f)
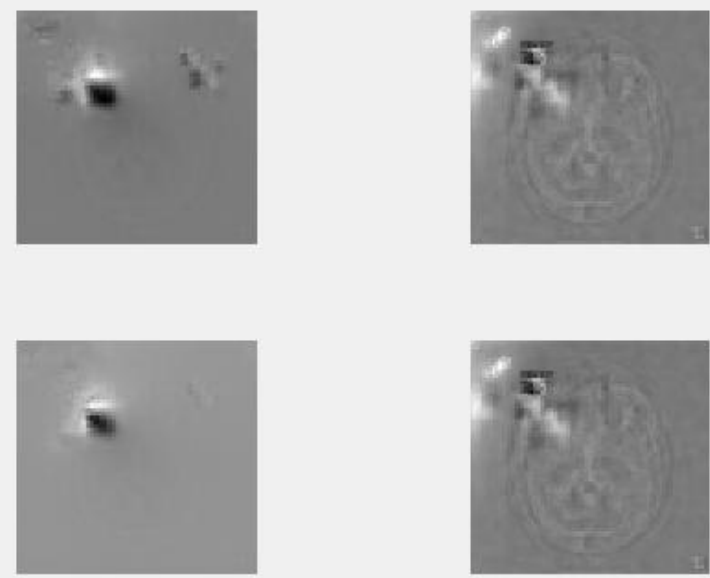

Figure 4(g)

Published By:

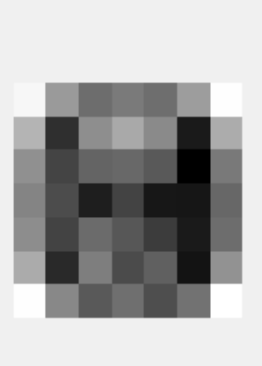

Figure 4(e)
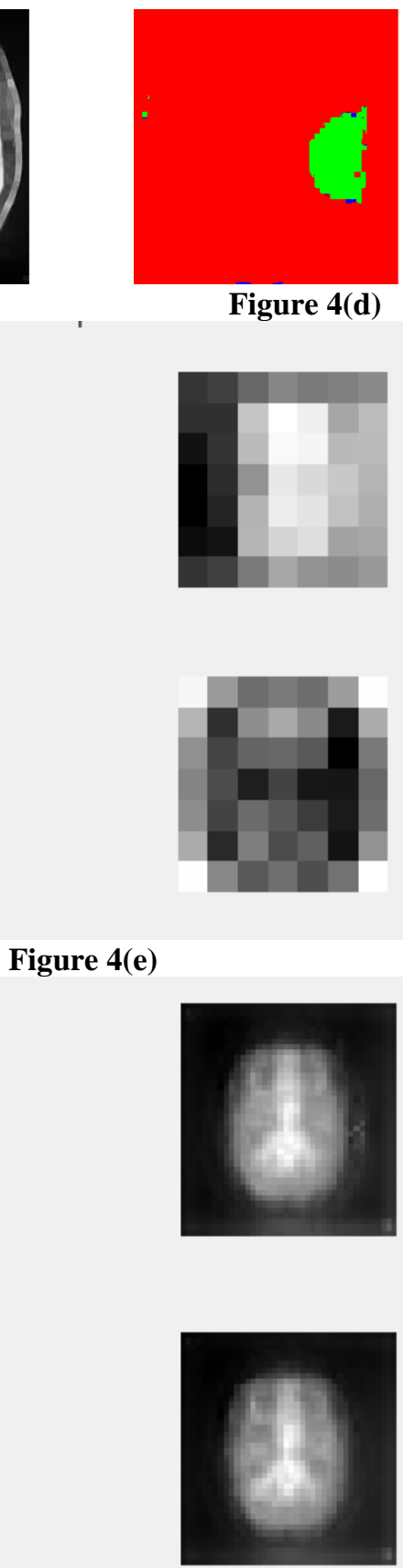

Figure 4(d)

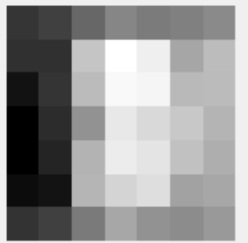



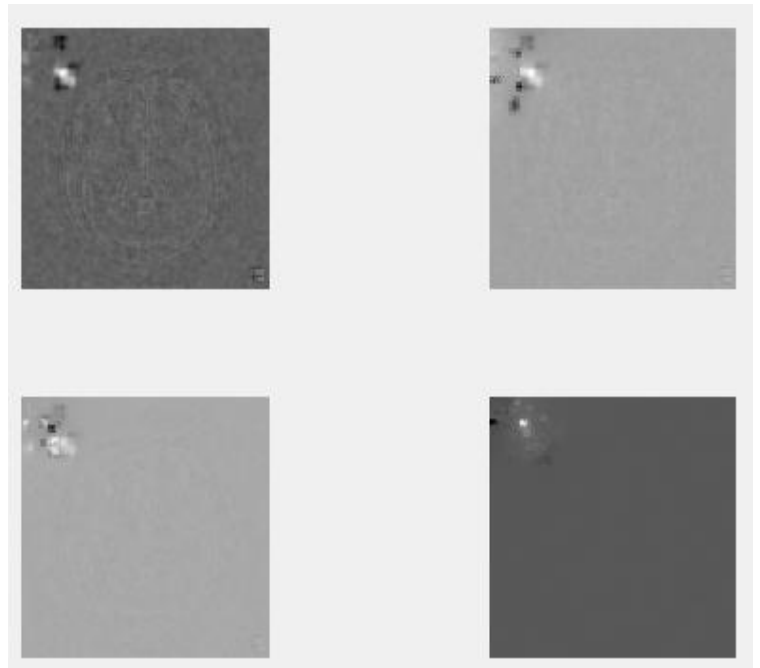

Figure 4(h)

Figure 4(a) Input image, 4(b) Pre-processed image, (c) Tumor section, 4(d) Six sigma segmentation output, 4(e) Layer convolution 1 features, 4(f) Layer convolution 2 features, 4(g) Layer convolution 3 features, 4(h) Layer convolution 4 features.

From Figure 5, it can be concluded in validation stage that, out of 30 normal brain images 28 images are correctly classified as normal brain, out of 30 Meningiomas images all 30 images are correctly classified as Meningiomas, out of 30 Gliobastoma Multiforme images all 30 images are correctly classified as Gliobastoma Multiforme, out of 30 Astrocytomas images all 30 images are correctly classified as Astrocytomas, out of 30 Pitutary tumors images 29 images are correctly classified as Pitutary tumors.

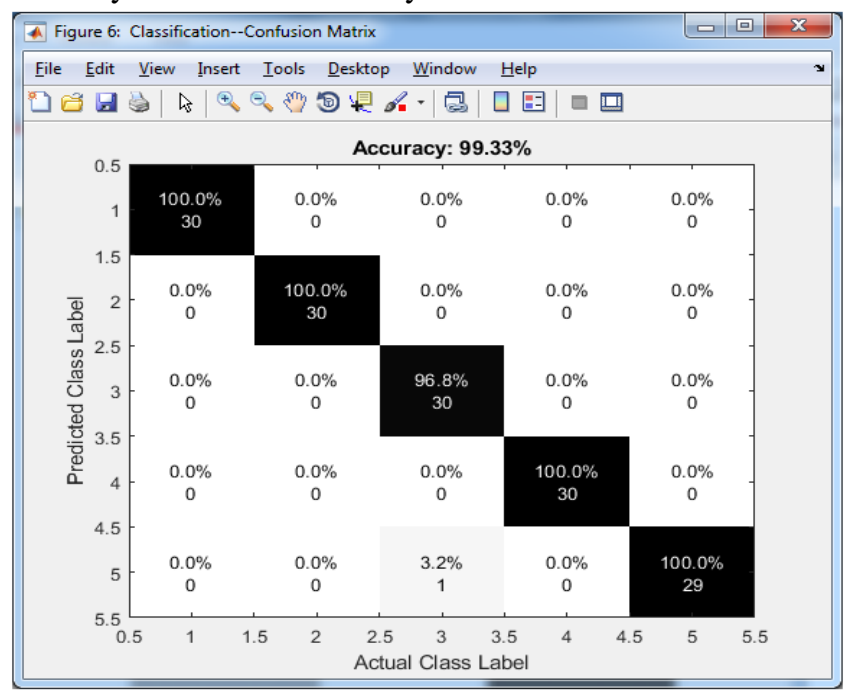

Figure 5: Resultant Accuracy chart

Each row of Matrix indicates instances in a predicted class while each column indicates instances in an actual class. Table 1; depicts the confusion matrix for the proposed system. Totally 500 brain MRI images has been taken for the evaluation. 350 samples used for training and 150 samples used for the testing. The results achieved $496 \mathrm{TP}$ rate, $2 \mathrm{TN}$ rate, $1 \mathrm{FP}$ rate and $1 \mathrm{FN}$ rate. The various parameters will be calculated based on the confusion matrix as shown below.

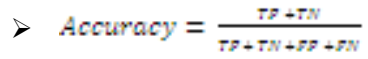

$$
\begin{aligned}
& >\text { Precision }=\frac{\pi B}{\pi p+F} \\
& >\text { Sensitivity }=\frac{M F}{\mathrm{TF}} \\
& >\text { Specificity }=\frac{\pi w}{\pi w+3 P}
\end{aligned}
$$

Performance will be measured by confusion matrix. Confusion matrix can also be known as error matrix is a specific layout which consents visualization of performance

\begin{tabular}{|c|c|c|c|}
\hline & $\begin{array}{c}\text { True Negative } \\
\text { (TN) }\end{array}$ & $\begin{array}{c}\text { True } \\
\text { Positive(TP) }\end{array}$ & Total \\
\hline $\begin{array}{l}\text { False Negative } \\
\qquad(\mathrm{FN})\end{array}$ & $\mathrm{TN}=2$ & $\mathrm{FP}=1$ & 3 \\
\hline $\begin{array}{l}\text { False Positive } \\
\text { (FP) }\end{array}$ & $\mathrm{FN}=1$ & $\mathrm{TP}=496$ & 497 \\
\hline Total & 3 & 497 & 500 \\
\hline
\end{tabular}
of proposed model. It is also called matching matrix which is depicted in Table 1.

Table 1: Confusion Matrix

- True Positive (TP): Model correctly predicts as positive.

- True Negative (TN): Model correctly predicts as negative.

- False Positive (FP): Model incorrectly predicts as positive.

- False Negative (FN): Model incorrectly predicts as negative.

The performance analysis of the proposed model can be concluded through following Table 2. The proposed hybrid model using CNN-SVM achieves much higher accuracy than

\begin{tabular}{|c|c|c|c|}
\hline Methods & $\begin{array}{c}\text { Feature } \\
\text { Extraction }\end{array}$ & Classification & $\begin{array}{c}\text { Accuracy } \\
(\%)\end{array}$ \\
\hline $\mathrm{CNN}$ & $\mathrm{CNN}$ & $\mathrm{CNN}$ & 36 \\
\hline $\begin{array}{c}\text { Multiclass } \\
\text { SVM }\end{array}$ & HOG & SVM & 93.33 \\
\hline $\begin{array}{c}\text { Proposed } \\
\text { Method }\end{array}$ & $\mathrm{CNN}$ & SVM & 99.33 \\
\hline
\end{tabular}
the individual models using CNN and SVM separately.

Table 2: Comparison of Proposed Method with the Existing methods

The accuracy comparison graph for classification is given in Figure 6. The various parameters like precision, sensitivity and specificity graphs which achieves $99.79 \%, 99.79 \%$ and $66.66 \%$ respectively for proposed model is shown in the Figure 7. 


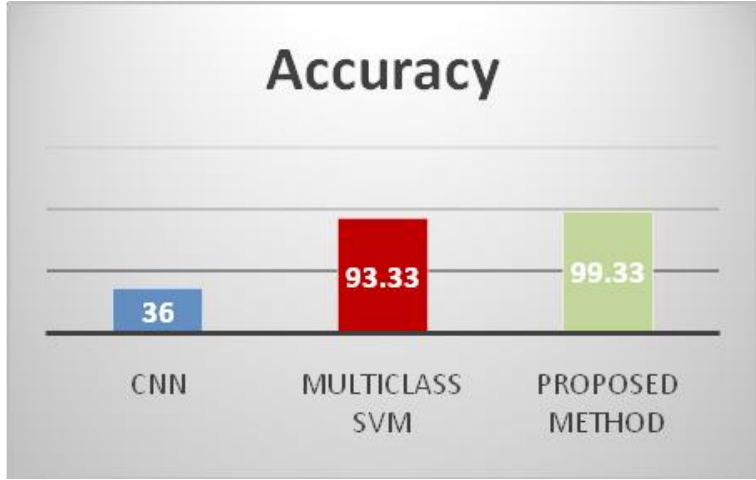

Figure 6: Accuracy Comparison Graph for Classification

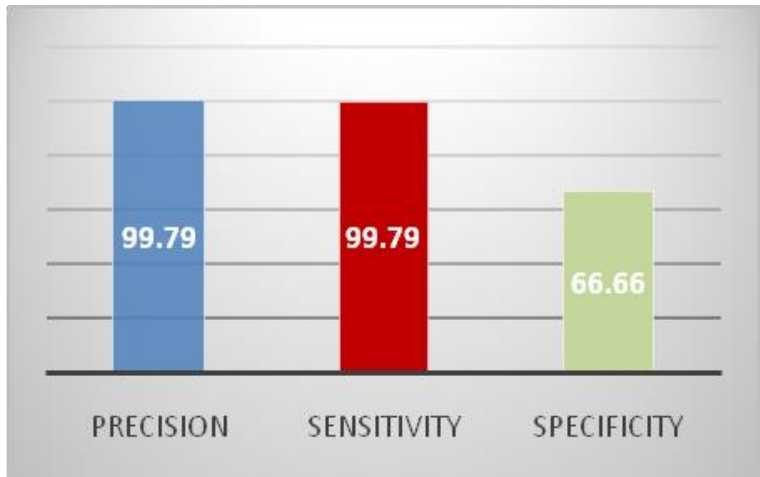

Figure 7: Precision, Sensitivity and Specificity Graph

\section{CONCLUSION}

Tumor identification and classification of Brain is still a challenging task due to numerous factors. The brain MRI tumor identification and classification has been proposed in this research work. The system is implemented to classify brain MRI image as normal and abnormal brain using supervised hybrid CNN-SVM model. The initial pre-processing has been done with the help of histogram equalization. Now the pre-processed input MRI is segmented using six sigma techniques. The key features extracted from CNN. The feature vector obtained is given as input to the SVM classifier. The classification of tumor is categorized into normal brain, meningioma, gliobastoma multiforme, astrocytoma and pituitary tumors has performed. The proposed system was implemented using MATLAB 2018a. The result shows the $99.33 \%$ of classification accuracy for majority of input data.

\section{ACKNOWLEDGEMENT}

This research paper is made through the help and support from database team. The authors are highly in-depth to the MRMC, Department of Radiology team, Kalaburagi, for extending support by providing the MRI imageries to carry out the research work.

\section{REFERENCES}

1. Gupta T, Gandhi T.K, Gupta R.K and Panigrahi B.K, "Classification of Patients with Tumor using MR FLAIR Images", Elsevier, Pattern Recognition Letters, 2017

2. Mohsen H, El-Dahshan E.S.A, El-Horbaty E.S.M and Salem A.B.M "Classification using Deep Learning Neural Networks for Brain Tumors" Elsevier, Future Computing and Informatics Journal, Vol. 3, No. 1, pp. 68-71, 2018.

3. Havaei M, Larochelle H, Poulin P and Jodoin P.M, "Within-Brain Classification for Brain Tumor Segmentation", Springer, International journal of computer assisted radiology and surgery, Vol. 11, No. 5, pp. 777-788, 2016.
4. Praveen G.B and Agrawal A, "Hybrid Approach for Brain Tumor Detection and Classification in Magnetic Resonance Images", IEEE, pp. 162-166, 2015.

5. [5] Wasule V and Sonar P, "Classification of Brain MRI using SVM and KNN Classifier”, IEEE, pp. 218-223, 2017.

6. [6] Nandpuru H.B, Salankar S.S and Bora V.R, "MRI Brain Cancer Classification using Support Vector Machine” IEEE, pp. 1-6, 2014.

7. [7] Usman $\mathrm{K}$ and Rajpoot $\mathrm{K}$, "Brain Tumor Classification from Multi-Modality MRI using Wavelets and Machine Learning”, Springer, Pattern Analysis and Applications, Vol. 20, No. 3, pp. 871-881, 2017.

8. [8] Raju A.R, Suresh P and Rao R.R, "Bayesian HCS-Based Multi-SVNN: A Classification Approach for Brain Tumor Segmentation and Classification using Bayesian Fuzzy Clustering", Elsevier, Biocybernetics and Biomedical Engineering, 2018.

9. [9] Sethumadhavan G and Sankaran S, "Segmentation using Six Sigma Threshold on Spectral Bands of Malignant Melanoma", In Proceedings of the International Multi Conference of Engineers and Computer Scientists, Vol. 1, pp. 18-20, 2009.

10. Danti A, "Segmentation and Classification of Raw Arecanuts Based on Three Sigma Control Limits", Elsevier, Vol. 4, pp. 215-219, 2012.

11. Havaei M, Jodoin P.M and Larochelle H, "Efficient Interactive Brain Tumor Segmentation as Within-Brain KNN Classification”, IEEE, pp 556-561, 2014

12. Havaei M, Davy A, Warde-Farley D, Biard A, Courville A, Bengio, Y, Pal, C, Jodoin, P.M and Larochelle H, "Brain Tumor Segmentation with Deep Neural Networks", Elsevier, Medical image analysis, Vol. 35, pp. 18-31, 2017.

13. Anitha V and Murugavalli S, "Brain Tumour Classification using Two-Tier Classifier with Adaptive Segmentation Technique", IET computer vision, Vol. 10, No. 1, pp. 9-17, 2016.

14. Sachdeva J, Kumar V, Gupta I, Khandelwal N and Ahuja C.K, "A Package-SFERCB-"Segmentation, Feature Extraction, Reduction and Classification Analysis by both SVM and ANN for Brain Tumors", Elsevier, Applied Soft Computing, Vol. 47, pp.151-167, 2016.

15. Machhale K, Nandpuru H.B, Kapur V and Kosta L, "MRI Brain Cancer Classification using Hybrid Classifier (SVM-KNN)" IEEE, pp. 60-65, 2015.

16. Mathew, A.R., Anto, P.B. and Thara, N.K., 2017, July. Brain tumor segmentation and classification using DWT, Gabour wavelet and GLCM. In Intelligent Computing, Instrumentation and Control Technologies (ICICICT), 2017 International Conference on (pp 1744-1750). IEEE.

17. Bento M, Sym Y, Frayne R, Lotufo R and Rittner L, "Probabilistic Segmentation of Brain White Matter Lesions using Texture-Based Classification", Springer, pp. 71-78, 2017.

\section{AUTHORS PROFILE}

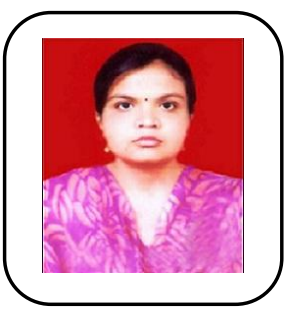

Arati Kothari received her M.C.A. degree from Department of Computer Science, Gulbarga University, Gulbarga, Karnataka, India in 2009. She is currently a PhD Scholar from Bharathiar University, Coimbatore, Tamil Nadu, India. Her research interests include Medical Image Processing, Digital Image Processing and Networking. She published 6 research articles in national and international journals.

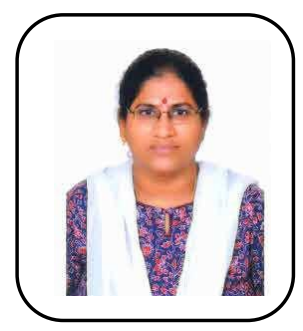

Dr. Baddam Indira received her MCA from Kakatiya University, Telangana in 1996 and a Ph.D. from Sri. Padmavati Mahila Visvavidyalayam, Tirupati, India in 2008. She is currently an assistant professor in Chaitanya Bharathi Institute of Technology, Hyderabad, India. She also has an over 22 years teaching experience. Her research interests include Digital Image Processing, Neural Networks, Data Structures and Cloud Computing.

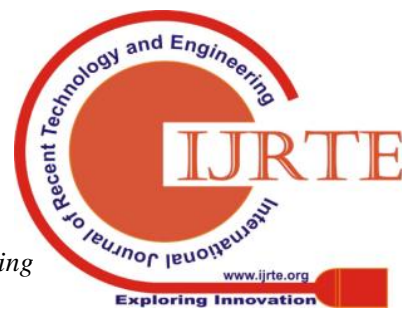

